\title{
The Application of Task-based Language Teaching to English Reading Classroom
}

\author{
Zan Mao \\ English Department, Zhenjiang Watercraft College of PLA, Zhenjiang, China \\ Email: shmaozan@sina.com
}

\begin{abstract}
Task-based approach is a new approach in education practice. In task-based classroom, the chief focus is the performance of tasks. The task, rather than being a unit of grammar to be digested or a collection of lexical items to be remembered, is a means of learning the language by doing tasks. It requires exposure to comprehensive input and opportunities to interact in the language. The task has to be negotiated by the learners with the help of the teacher, whose job is to facilitate learning opportunities in class. The task probably needs the cooperation of learners. This paper is intended to explore TBLT in teaching reading on the basis of the notion of task-based language teaching (TBLT).
\end{abstract}

Index Terms — task, task-based language teaching, task-based approach, English reading classroom

\section{INTRODUCTION}

Task-based language teaching (TBLT) has been strongly advocated and promoted by many world-leading linguists (Long 1985, Prabhu 1987, Nunan 1989, Willis 1996, Skehan 1998, Bygate \&Ellis 2001) since early 1980s. Despite differences they may hold, they all emphasize that language teaching should interact with learning to create genuine use of the language, and that language proficiency can be achieved by doing tasks. And in 2001, the Ministry of Education issued the new National Curriculum Standards for English education and suggested adopting TBLT in English teaching in schools. That is "To sense, experience, practice and cooperate under the teacher's instruction aiming to reach the goal of tasks".

TBLT has been applied by English teachers of China gradually, especially in the reading classroom for being able to read is critical in contemporary life. Reading is an active process, during which the reader tries to understand the meaning of a given text. By sensing, experiencing, practicing and cooperating, learners can understand the meaning of the text more easily. However, the present situation of reading in China is not satisfactory, because many teachers are still using teacher-centered methods, which do not attach enough importance to the improvement of learners' communicative competence. And although task-based approach (TBA) has been tried by many English teachers, it remains a new thing to most teachers, especially to the teachers who adopt traditional teaching method. Therefore, it is high time to change this situation by adopting a TBLT approach to English reading classroom so that the satisfactory result can be achieved.

This paper attempts to illustrate that TBLT can be feasible and is an effective teaching approach for English reading classroom. It will also introduce the research of TBLT by distinguished linguists and some new view on how to apply TBLT to English reading classroom.

\section{LITERATURE REVIEW}

\section{A. Definition of "Task"}

Numerous linguists around the world have attempted to define the term "task" as it relates to task-based language teaching (TBLT) since the 1980s. If we look in the literature, we can find that task is variously defined. Long (1985) gives the definition in a broad sense:

A task is a piece of work undertaken for oneself or for others, freely or for some reward. Thus, examples of tasks include painting a fence, dressing a child, filling out a form, buying a pair of shoes, making an airline reservation, borrowing a library book, etc. In other words, by 'task' is meant the hundred and one things people do in everyday life, at work, at play and in between. (p.89)

This is a non-pedagogical definition in that it describes the sorts of things individuals do outside the classroom, and some of these tasks do not necessarily involve the use of language.

Richards, Platt \& Weber (1986) offered a more pedagogically oriented definition: "task is an activity or action which is carried out as the result of processing or understanding the language" (p.289). For example, drawing a map while listening to a tape, listening to an instruction and performing a command, may be referred to as tasks.

This definition implies that tasks involve communicative language use in which the user's attention is focused on meaning rather than linguistics structure. David Nunan's definition of task reflects the characteristic. He stressed meaning for the first time: "Task is a piece of classroom work which involves learners in comprehending, manipulating, 
producing or interacting in the target language while their attention is principally focused on meaning rather than on form" (Nunan, 1989, p.10).

Peter Skehan (1998) in his book A Cognitive Approach to Language Learning lays a solid foundation for defining a task from a pedagogical perspective by reflecting a broad consensus among researchers and educators. He suggests five defining criteria: A task is an activity in which a) meaning is primary b) there is some communication problem to solve c) there is some sort of relationship to comparable real-world activities d) task completion has some priority e) the assessment of the task is in terms of outcomes.

The latest development in TBLT is the work done by Martin Bygate. In his Researching Pedagogic Tasks (Applied Linguistics and Language Study), Martin Bygate (2001) points out that a task is an activity which requires learners to use language, with emphasis on meaning, to attain an objective, and that the central challenge for language teaching is to develop learner' communicative language ability through pedagogic intervention with tasks accomplished.

Richards, Platt and Weber's definition, Nunan's definition and Bygate's definition of task are all pedagogical. Richards, Platt and Weber's is not so comprehensive as the latter ones. Both of the latter ones emphasize on meaning, and Nunan's focuses on the actions of doing a task, while Bygate's stresses the communication. Skehan's criteria cover all the aspects of a task.

Combining Nunan's and Bygate's definition with Skehan's criteria, I give my definition of "task": Pedagogically, a task is a classroom activity which involves learners with the help of the teacher in comprehending, manipulating, producing or interacting in the target language with a communicative goal. According to this definition, I define the "task" in English reading classroom as a reading classroom activity which involves learners with the help of the teacher in comprehending the reading material, manipulating the language usage in the material, producing their own language output based on the comprehension of the usage, or interacting with each other about their opinion related to the material in English with a communicative goal.

\section{B. Task Components}

According to Nunan (1989), the definition of a language-learning task requires specification of six components: goals, input, activities, teacher role, learner role and settings.

1. Goals

Goals are the vague general intentions behind any given learning task.

Goals provide a point of contact between the task and the broader curriculum. As to it, a teacher may be asked that why he/she got learners to engage in Task X. The answer will general take the form of some sort of goal statement. Possible answers might be:

"I wanted to develop their confidence in speaking."

"I wanted to develop their personal writing skills."

"I wanted to encourage them to negotiate information between each other to develop their interactional skills."

"I wanted to develop their reading comprehension skills."

Goals may relate to a range of general outcomes (communicative, affective or cognitive) or may directly describe teacher or learner behavior. And goals are not always explicitly stated, although they can usually be inferred from an examination of a task. In addition there is rarely a simple one-to-one relationship between goals and tasks. In some cases a complex task involving a range of activities might be simultaneously moving learners towards several goals. In the teaching of reading, goals may be to get the learners to learn the topic, or find the detailed information or get the main idea of the reading material, etc.

2. Input

Input refers to the data that form the point of departure for the task. In fact, input for communicative tasks can be derived from a wide range of sources. Hover (1986) suggests a list of sources, to name just a few, letters, newspaper extracts, memo note, shopping lists, recipe, weather forecast, etc.

Quite a few pedagogues advocate using authentic materials as the sources of tasks. "Authentic" here is any material that has not been specifically produced for the purposes of language teaching. Proponents of authentic materials point out that classroom texts and dialogues do not adequately prepare learners for coping with the language they read in the real world outside the classroom. They argue that if we want learners to comprehend written texts in the real world, then the learners need opportunities for engaging in these real-world texts in class.

Hover's list of sources of input is quite authentic, which accords with Skehan's criteria: task has some connections with real-world activities. The aim to learn a language is to use it in the real world, so using authentic materials in the reading task is quite necessary.

3. Activities

Activities specify what learners will actually do with the input which forms the point of departure for the learning task. Nunan (1989) proposes three general ways of characterizing activities: rehearsal for the real world, skills use, and fluency/accuracy.

We shall now look at some proposals relating the development of reading skills.

Grellet (1981) provides the following typology for reading comprehension activities:

Activities in reading techniques are designed to develop basic reading skills and strategies. Learners are encouraged to increase their reading speed, and to develop flexible reading strategies (in particular, scanning for specific 
information, and skimming for an overview of the text).

The activities focus the learner on how writers convey their aim through the function and organization of the text. Learners are taught to identify the function of the text by utilizing linguistic and non-linguistic clues. They are also taught to identify the essential organization of the text, whether it is through the expansion of a main idea, and/or whether it is organized in terms of chronological sequence, description, analogy and contrast, classification, argument and logic. Finally, learners are sensitized to the mechanics of schematization. They are shown how altering the order of elements in a sentence can alter the meaning.

Activities which focus on meaning are designed to get learners to process the content of the text through the various types of non-linguistic and linguistic responses they might make to the text. The activities have two different aims:

1). To make students active in the reading process by presenting them with decision-making activities (e.g. drawing a diagram with the information given in the text, solving the problem, completing a table which reorganizes the information).

2). To devise activities which are as natural as possible, i.e. as close as possible to what one would naturally do with the text (e.g. answering a letter using the information given in that letter, completing a document, comparing several texts, etc.)

Activities focusing on assessing the text are designed to get readers to go beneath the surface of the text, as it were, in order to judge it and evaluate it. Here readers are required to differentiate fact from opinion and to identify the writer's attitudes, intentions and biases.

Grellet's typology is comprehensive. It analyzes the reading process in terms of the cognitive demands made on the reader. Some of the elements relate to reading techniques, for example skimming and scanning, which are essential skills to comprehend the given text. Some relate to rhetorical functions, such as chronological sequence and classification, which are the basis to learn the organization of the text. Some relate to cognitive operations such as understanding relations within the sentence, which are helpful to understand the detailed information and the structure. Others relate to classroom tasks such as jigsaw reading and reorganizing information using grids, which can help to read more actively. The teacher should teach students to read from these aspects so as to reach the aim of reading effectively.

4. Teacher's and learners' roles

"Role" refers to the part that learners and teachers are expected to play in the carrying out learning tasks as well as the social and interpersonal relationships between the participants (Nunan, 1989).

Learner roles are closely related to the functions and status of the teacher (Richards and Rodgers, 1986). Giving the learners a different role, such as greater initiative in the classroom, requires the teacher to adopt a different role. The teacher is no longer a passive recipient and implementer of other people's syllabus and methods but an active creator of his or her own materials, classroom activities and so on.

According to Breen and Candlin (1980), the teacher has three main roles in the communicative classroom. The first is to act as facilitator of the communicative process, the second is to act as a participant, and the third is to act as an observer and learner.

As to me, the teacher should first be the facilitator of the reading material and the designer of the task. Second, the teacher should be the observer and guide. Learners may be confused about what they should do and how they can read during the task, so the teacher should monitor the reading process of learners so as to give the timely guidance, and observe carefully at the performance of learners so as to praise them or give them the suggestion of improvement. Finally, the teacher should be a listener and learner. Learners are usually creative, so the teacher should listen to their opinions, exchange his/her idea with them and maybe he/she could learn from them, which is called that teaching benefits teachers as well as learners.

Reading, in many other people's view, is a boring work. However, in my point of view, it is an active process, during which the reader tries to understand the meaning of a given text. With the guidance of the teacher, learners can understand the meaning more effectively and actively.

5. Settings

"Settings" refers to the classroom arrangements specified or implied in the task, and it also requires consideration of whether the task is to be carried out wholly or partly outside the classroom. Settings will be an important factor influencing roles and relationships. Whether the task is on individual, pair, group, or the whole class basis decides the relationship between learners or between the teacher and learners. As I mentioned in 2.2.4, reading as an active process, it needs the needs the thinking of the individual or it needs the negotiation and cooperation of learners, so the task can be arranged in individual, pair, group, or even the whole class according to the needs.

\section{Types of Task}

1. Willis' classification

As classified by Willis, there are six main types of task adapted for use with almost any topic from the aspect of the actual use of language.

Listing:

Listing may seem unimaginative, but in practice, listing tasks tend to get a lot to talk as learners explain their ideas. The types involved brainstorming, in which learners draw on their own knowledge and experience either as a class or in pairs/groups; act-finding, in which learners find things out by asking each other or other people and referring to books, 
etc.

Ordering and sorting:

Ordering and sorting tasks involve four main types: sequencing items, actions or events in a logical or chronological order; ranking items, according to personal values or specified criteria; categorizing items in given groups or grouping them under given headings; classifying items in different ways, where the categories themselves are not given.

Comparing:

Broadly, comparing tasks involve comparing information of a similar nature but from different sources or versions in order to identify common points and/or differences.

The processes involved matching to identify specific points and relate them to each other; finding similarities and things in common; finding differences.

Problem solving:

Problem-solving tasks make demands upon people's intellectual and reasoning powers, and though challenging, they are engaging and often satisfying to solve. The processes and time scale will vary enormously depending in the type and complexity of the problem. Real-life problems may involve expressing hypotheses, describing experiences, comparing alternatives and evaluating and agreeing a solution. Completion tasks are often based on short extracts from texts, where the learners predict the ending or piece together clues to guess it. The classification ends with case studies, which are more complex, entail an in-depth consideration of many criteria, and often involve additional fact-finding and investigating.

Sharing personal experiences:

Tasks of sharing personal experiences encourage learners to talk more freely about themselves and share their experiences with others. For example, after reading a selected material about one's childhood, learners can be encouraged to tell their own childhood. The resulting interaction is closer to casual social conversation in that it is not as directly goal-oriented as in other tasks. For that very reason, however, these open tasks may be more difficult to get going in the classroom.

Creative tasks:

These are often called projects and involve pairs or groups of learners in some kind of freer creative work. They also tend to have more stages than other tasks and can involve combinations of task types above. Out-of-class research is sometimes needed. Organizational skills and teamwork are important in getting the task done. The outcome can often be appreciated by a wider audience than the students who produced it.

2. Prabhu's classification

Prabhu identifies three kinds of cognitive task types: information-gap, opinion-gap and reasoning-gap tasks.

An information-gap activity involves the exchange of information among participants in order to complete a task. For example, an information-gap activity might involve a student describing a picture for another student to draw or students drawing each others' family trees after sharing information.

An opinion-gap activity requires that students give their personal preferences, feelings, or attitudes in order to complete a task. For instance, students might be given a social problem, such as high unemployment and be asked to come up with a series of possible solutions. Another task might be to compose a letter of advice to a friend who has sought their counsel about a dilemma.

A reasoning-gap activity requires students to derive some new information by inferring it from information they have been given. For example, students might be given a railroad timetable and asked to work out the best route to get from one particular city to another or they might be asked to solve a riddle. Prabhu (1987) feels that reasoning-gap tasks work best since information-gap tasks often require a single step transfer of information, rather than sustained negotiation, and opinion-gap tasks tend to be rather open-ended. Reasoning-gap tasks, on the other hand, encourage a more sustained engagement with meaning, thought they are still characterized by a somewhat predictable use of language.

3. Closed and open tasks

From the aspect of teaching methodology and the practice of learners, tasks can also be divided into closed tasks and open tasks. "Closed tasks are ones that are highly structured and have very specific goals. Open tasks are ones that more loosely structured, with a less specific goal" (Willis, 1996, p.28). As to reading, working in pairs to compare the difference of topic between two paragraphs is considered a closed task, because the instruction and the information are much tightened. Whereas for the open task, one typical example is to express one's own opinion about the topic of the chosen text, for this is quite up to the participants' own way of task-completion and much personal perspective is added, and there is no definite outcome for the task.

Besides these closed and open tasks, there are also some tasks coming midway between the two, such as problem-solving task or ranking tasks which have specific goals. But they could be approached from different ways. No matter what task is engaged in the learning process, the ultimate goal is to create an effective learning environment in the classroom to meet three essential conditions: the provision of exposure to the target language, the provision of opportunities for learners to use the target language for real communication and the provision of motivation for learners to engage in the learning process.

Willis' classification is more specific, Prabhu's more abstract, and the classification of closed and open tasks is the most general. In Willis' classification, the first three one, listing, ordering and sorting and comparing belong to closed 
tasks, while problem solving, sharing personal experiences and creative tasks belong to open tasks. As to Prabhu's, information-gap activities belong to closed ones, opinion-gap activities belong to open ones, and reasoning-gap activities to the ones between the closed and the open ones. To classify tasks based on the characteristics, contents and the ways of doing of TBLT will help teacher adopt different teaching modes according to different learners, different reading tasks and different reading stages, and will also help promote English reading teaching and learning efficiency.

\section{Task-based Approach (TBA) VS Traditional Teaching Methods}

The traditional methods have dominated English language teaching in China for decades. The traditional methods focus on form and are featured as teacher-centered, such as language-point approach and sentence-text-analysis approach. After the birth of task-based approach (TBA), traditional methods began to show its deficiency.

First, TBA emphasizes on meaningful communication as well as form and the cooperation between learners and the teacher, as has reflected from the meaning of "task" (2.1) and Prabhu's classification (2.3.2). As a contrast, traditional methods focus on form and are featured as teacher-centered, as has mentioned above. Gray (1990) points out a brief comparison between traditional methodology (TM) and TBA: meaningful communication is limited in TM, while meaningful communication as key feature in TBA; the teacher is the decision-maker in TM, while the learner and the teacher as the joint decision-maker in TBA.

Second, just like Willis' classification, tasks are all closely related to the real life, such as problem solving and sharing personal experiences, while TM always focuses on mechanical grammar exercise. In my experience of being a student and an intern, the traditional classroom is deadly dull, which is full of drills and drudgery, while the task-based classroom is a much more exciting place, with students actively involved on tasks and topics, which are stimulating and motivating for learners. TM focuses on learning rules, grammar and word-formation, while TBA on language awareness (Gray, 1990).

As to reading, readers try to understand the meaning of the given text. Therefore, in this view, meaning is more important than form in reading. And the reason we read is to enhance our reading ability to read the various kinds of information in our daily life, and tasks are closely related to real life, so TBA can develop our reading skills more effectively.

\section{A Shift From Traditional TEACHING Methods to TBLT}

\section{A. Current Situation of English Reading Classroom in China}

As we all know, reading in English is an essential comprehensive language course. The aim is to enhance students' language proficiency and develop the all-round competence. In China, for a long time reading has been playing an important role in English classroom.

In order to find out relative information concerning reading activity, a questionnaire was designed. The subjects of this questionnaire were 50 students of Class 5 Grade 2 of Shanghai No.4 Middle School. After the papers were collected, the data were calculated and analyzed, and some problems of English reading classroom were found out.

The first question is about the reasons for students to do reading. This is a multiple-choice question. The aim is to see the students' purpose of reading, and how much interest they have in reading. The chosen items, the number of each item the students choose and the percentage are in the Table 1.

\begin{tabular}{l} 
TABLE 1: \\
\begin{tabular}{|l|l|l|}
\hline 1) Why do you want to do reading? & number & percentage \\
\hline Chosen item & 14 & $28 \%$ \\
\hline for more information & 6 & $12 \%$ \\
\hline for the pleasure of reading & 16 & $32 \%$ \\
\hline for examination purpose & 20 & $40 \%$ \\
\hline for possible future job &
\end{tabular} \\
\hline
\end{tabular}

Only 6 out of 50 students read for pleasure while 16 for examination purpose and 20 for the preparation of future job. The motivation of most students is to achieve a better school report so as to make a full preparation for the possible future jobs. As a result, little pleasure and delight can be attained from reading. This instrumental motivation may be overpowered by test and by emphasis in academic achievement rather than personal capability. This is mostly because the education system is still test-oriented, and it results that the reading class is not related to the real life, while, according to Skehan, one of the criteria of task is that there is some sort of relationship to comparable real-world activities.

The second question is on the basis of the first question. The first one affects the result of the second one. The second one is about the students' requirement to reading materials which include interest in reading materials, information contained in materials, degree of difficulty, length of materials and the new vocabulary involved in materials. Each of them is divided into three degrees. The chosen items, the number of each item the students choose and the percentage are in the Table 2. 
TABLE 2:

\begin{tabular}{|l|l|l|l|l|l|l|l|l|l|}
\hline 2) Your requirement to reading materials & great & 46 & $\mathbf{9 2 \%}$ & medium & 3 & $6 \%$ & less & 1 & $2 \%$ \\
\hline interest & rich & 11 & $22 \%$ & medium & 32 & $\mathbf{6 4 \%}$ & less & 7 & $14 \%$ \\
\hline information & difficult & 6 & $12 \%$ & medium & 43 & $\underline{86 \%}$ & easy & 1 & $2 \%$ \\
\hline degree of difficulty & long & 3 & $6 \%$ & medium & 32 & $\underline{64 \%}$ & short & 15 & $30 \%$ \\
\hline length & large & 3 & $\underline{6 \%}$ & medium & 24 & $48 \%$ & less & 23 & $46 \%$ \\
\hline new vocabulary &
\end{tabular}

The data in boldface show that a majority of students prefer stories and articles which can arouse their great interests with appropriate amount of information inside. The students generally find themselves in a frustrating position to deal with a cloud of information without any advice or guidance from the teacher. The characteristic of task is much about the real life, and TBLT emphasizes on "Learning by doing" (Nunan, 1989), which is closed to the real life, and can arouse learners' interest. According to the underlined data, a number of students refuse the materials with enormous new vocabulary. They tend to choose reading materials with a comparative easier understanding and appropriate length.

The third question is about the activities students usually do in English reading class. It is a multiple-choice question. The aim is to see the way their teacher gives the reading lesson and the attitude of students having the reading lesson. The chosen items, the number of each item the students choose and the percentage are in the Table 3 .

TABLE 3:

\begin{tabular}{|l|l|l|}
\hline 3) What do you usually do in English reading class? \\
\hline Chosen item & number & percentage \\
\hline listening to the teacher and taking notes & 33 & $66 \%$ \\
\hline listening to others' statement or speech & 12 & $24 \%$ \\
\hline answering many questions raised by teacher & 6 & $12 \%$ \\
\hline various topic discussion & 10 & $20 \%$ \\
\hline
\end{tabular}

In English reading classes, as many as $66 \%$ of the students are accustomed to listening to the teacher attentively followed by serious note-taking, which throw themselves into passively waiting the ready-made answers. Some students are lazy and unwilling to participate in the task in the group and completely depend on others. Though a number of the students are in favour of various discussions about the reading topic, they may show a slow and inactive reaction to the questions that the teacher puts forward without an appropriate guiding activity.

The fourth question is about the teacher's focus in reading classroom, i.e. whether the teacher's focus on grammar or meaning and whether on the interaction. The chosen items, the number of each item the students choose and the percentage are in the Table 4.

TABLE 4:

\begin{tabular}{|l|l|l|}
\hline 4) How does the teacher teach you reading? & number & percentage \\
\hline Chosen item & 32 & $64 \%$ \\
\hline (1)focus on grammatical rules & 18 & $36 \%$ \\
\hline focus on meaning & 16 & $32 \%$ \\
\hline (2)considerable interaction in classes & 34 & $68 \%$ \\
\hline limited interaction in classes & \\
\hline
\end{tabular}

The fact that $64 \%$ teachers focus on grammatical rules and $68 \%$ teachers have limited interaction in classes shows that most English classes are teacher-dominated with the explanation of grammatical forms rather than meaningful communication. The interactions between the teacher and students and between the students themselves are quite limited, which produces an obstacle in stimulating and motivating for learners. However, TBLT puts much emphasis on interaction, as the definition of "task", learners should interact in the target language with a communicative goal.

To conclude, most students land themselves in a passive position in reading class. To some degree, traditional teaching methods which have dominated the English classes for a long time has prevented the students from developing their reading interest and competence. As a result, a vicious circle is produced. It should be taken into account that how to change the situation by transforming the teaching approach in a positive way.

The last question is an open question about the problems students found when they were reading. This paper gives some typical cases of the students' problem.

5) Problems found in reading

Student A: Each time I meet with some unknown words, I become puzzled at them and slow to get into the state, which prevents me from getting a better understanding to the paragraphs, especially when I make a wrong guess.

The biggest problem of many students such as Student A is unfamiliar with vocabularies which hinder them from coherent comprehension to reading. Although some words look familiar, they may fail to recall their meanings immediately.

Student B: I want to speed up my reading, but the problem is that whenever I try it, I achieve almost nothing valuable from reading because my capacity for understanding is not fast enough. When time is passing, I feel a great pressure and can hardly concentrate well again.

Some students such as student B describe that when they are trying to develop the speed by scanning in the beginning, 
they can not equally grasp useful information fast enough.

Student C: Sometimes I can hardly connect those vocabulary and sentences into a meaning association pinpointing each paragraph and the whole passage. And I'm always slow in reacting with those logical thinking where I find my mind goes nowhere.

Many students such as student $\mathrm{C}$ report that it is sometimes hard to put all the words and sentences they have read into a meaning association. Therefore, they are slow to figure out the key points for each paragraph, especially unable to unfold the logical meaning in the context.

Student D: I am afraid of long sentences with complex structures. It's difficult to finger out their meanings without the teacher's instruction, but they often cover the key plots of the articles I'm reading.

Many other students such as student D have increased a fear for reading for lack of competence to deal with those complicated sentence structures where more than one clause might appear, which affects their comprehension to the whole passage.

Student E: There are often several meanings for some vocabulary, but I cannot recognize their extended meaning in the context provided. Maybe I need practice a lot in this aspect.

A number of students such as student E notice that they can catch the literal meaning but not the extended meaning of sentences. This is a utilization problem. As a result, they are unable to fill in the gaps with replaceable lexical items in a similar context.

Student F: When I do some reading, I find difficult to grasp the main idea of the passage. Let alone to refer or conclude something relative. What's worse, sometimes I go away from the writer's train of thought.

Some subjects such as student F are confused about the main idea of the passage or they can not even infer some relative information based on the original. Although they have successfully parsed some parts of the input, they still can not form a deeper thought and make correct choices on test papers.

Knowing the students problems in the course of reading will naturally place the teacher in a better position to guide learners in coping with or overcoming these difficulties. What the teacher should do is to improve teaching methods and open up a new prospect for teaching English reading. TBLT can make learners learn by doing tasks as what they do in real life. By this way, learners will have more interest in learning and are more easily to remember what they have learned.

\section{B. The Necessity of the Shift}

The new National Curriculum Standards for English Education suggested implementing TBLT in English teaching so as to do things with words and foster students' comprehensive competence of using the language. In this situation, it is the job of teachers to motivate students to learn, and to give them the confidence to succeed. It is to some extent a pedagogical shift in foreign language learning and teaching, from a more traditional, teacher-oriented and knowledge-based approach to a more interactive, communication-oriented, student-centered and task-based approach.

In running a reading class, we must take the following into consideration: What are the students' needs and abilities? How extensive are the materials and activities? How can they inject extrinsic motivation into a highly intrinsic situation? Such kind of ideas produces effective learning. Effective factors, such as confidence, motivation, attitudes to learning and anxiety, are more important to the learning process than cognition, and a confident, motivated student will learn much more easily and ably than one suffering from low self-confidence, low motivation, high anxiety, and a negative attitude to learning.

As we all know, to learn a language well, the intrinsic motivation of learners is a crucial factor. However, how to arouse learners' interest depends on the way the teacher teaches them. Today's English reading classrooms are still teacher-dominated, and most teachers focus on grammatical rules. This kind of way of teaching will make learners in a passive position in reading classroom, and hinder them improving communication ability.

However, if TBA is applied to reading classroom, the situation will be totally different. According to Gray (1990), TBA focuses on language awareness, not on grammatical rules, and many linguists advocate using authentic materials as the sources of tasks. Thus, learners can be put into an English Environment of the real use and can be encouraged to think in English rather than translate what they read into Chinese. And TBA needs the communication between the teacher and learners, just like what mentioned above, "learner and teacher as joint decision-maker in TBA" (Gray, 1990, p.78). In this situation, learners will have a feeling that they are involved in the class, and they are responsible to how they should read. And the "learning by doing" (Nunan, 1986, p.26) principle makes learners to acquire the knowledge by doing tasks rather than reading silently by themselves or listening to the teacher and taking notes. In this way, learners can feel the fun in reading, and they will be more interested in English.

In a word, adopting TBA is a good way to remedy the disadvantages of TM and it is a more effective way to learn English.

\section{Designing ReAding Class aCCORding to TBLT}

It is believed that the reason why most English teachers in China still adopt TM is that few task-based textbooks are published and even few are used in spite of TBLT is emphasized on greatly in task-based language teaching. Therefore, teachers have to design tasks for non-task-based materials to fit TBLT. Since TBLT is still a new mode for most of 
Chinese teachers, they may be lacking skills in designing and selecting appropriate tasks.

This study has collected many theories about TBLT and formed new views about it. Here, some viewpoints about how to design tasks for non-task-based materials in a reading lesson plan will be illustrated.

The experimental material for this study was chosen from Oxford English (S2A), the English textbook for students in grade 2 in senior high school in Shanghai, named "A practical joke". It is a story about a Mr. Potts being fooled by his colleagues. The colleagues changed between his hat and a hat of the same color but a bit larger. Finally, Mr. Potts felt so confused that he went to see the doctor. The whole text is attached in Appendix 1 while lesson plan of the first class is attached in Appendix 2.

The experimental class time is designed to divide into three stages: pre-reading stage, while-reading stage and post-reading stage. Before the lesson, some preparations should be made. The first step is to read the material and find out what is the main idea of the reading material and what elements should students get from the material. According to Grellet, the essential organization of the text should be identified first, so as to decide how to teach. This text is about a story which organized in chronological sequence, so tasks related to sequence is better to be assigned. The next step is to analyze the various tasks provided in the unit and discover if any of them can be used, or be adapted to be used, as a central communicative task. Then, a TBL framework is constructed around the central communicative task in order to help students learn the main idea. If there are no provided tasks can be used, some more qualified tasks should be designed by teachers themselves. Once a task or series of tasks have been adopted, a TBL framework around the chosen tasks should be constructed.

\section{A. Pre-reading Stage}

This is the first and primary stage of the teaching procedure, the aim of which is to activate the background knowledge of the readers, arouse their interest to the text and set up a purpose for them to read on. It is intended to provide readers with opportunities to activate their own existing schematic knowledge and to use their imagination to make predictions. This stage can serve as the preparation stage. The tasks given at this stage will usually enable the learners to engage in active purposeful interaction which will fan their desire to read the text. Students may be motivated and prepare themselves better for the reading task and understand more about the passage when they get into detailed study of it.

Since the title of the text is "A practical joke", some pictures and examples may be used to help students to understand the definition of "practical joke" at the beginning of the class. This is the background of this text and it can pave the way for the while-reading stage. Then, in order to find out whether the students acquire the term, teacher may ask them to tell some practical jokes they know or have experienced. This is a kind of open task what Willis called "sharing personal experience". The aims and specific procedures can be seen in Appendix 2.

\section{B. While-reading Stage}

At this stage, the teacher can design and assign some tasks to make students read the text efficiently and have a profound understanding. Tasks are designed to train students' reading skills such as scanning, skimming, reading for thorough comprehension and critical reading.

As for the reading activities, there are many forms that can be recommended in class: read for specific information; read for gist or general ideas of the text; deduce the meanings of certain words from a given context; infer the writer's intended message from a given context; recognize author's purpose and attitude.

In the experimental teaching plan, students are required to skimming the first two paragraphs and scanning some detailed information of the text so as to learn the topic of the text. After that, they are required to read the text and find out what happened to Mr. Potts's hat. Then, they are directed to discuss what they find in groups, and each group draws a chart of the changes of the "hat". Then do Exercise E3 ---- "Look at these pictures and sentences which show events from the story, and put the events in the correct time order in which they happened." At last, groups report their work. This task is a ordering task in Willis's classification and the reason for this design is that tasks based on text could motivate learners to read for a particular purpose. The sequence of the changes of the "hat" is the main thread of the story, so it is made a central communicative task.

\section{Post-reading Stage}

Post-reading activities are principally designed for learners to practice communicative output. The tasks at this stage are designed to offer students communicative opportunities to use the language points to communicate and extend the content of the text to the real-life situation. There are many forms available for the activities of the stage, such as text rewriting, role-playing, suggestion making, discussion, report, etc. these activities involve speaking, listening and writing.

At this stage, group or pair work, in my point of view, can make the reading more communicative. The students are at the center of the activities. They are active readers and participants in the reading tasks. The teacher is supposed to be an organizer and a guide.

At this stage, group or pair work can make the reading class more communicative. The students are at the center of the activities. They are active readers and participants in the reading tasks. The teacher is supposed to be an organizer and a guide. 
The ending of the text is that Mr. Potts went to see the doctor, and the doctor said to him, "Nonsense! There's no such kind of disease!" (more details can be found in Appendix 1). The text does not tell the reader what Mr. Potts said to the doctor, so a prediction task is designed. Students should speculate the scene of Mr. Potts' seeing the doctor, and do a role-play about it in pairs. One acts as Mr. Potts, who is required to guess Mr. Potts would describe his puzzle to the doctor according to the text. The other acts as the doctor, who is to think what kind of suggestion the doctor would give Mr. Potts (instruction can be found in Appendix 2).

This kind of prediction tasks belong to reasoning-gap ones classified by Prabhu, which are still characterized by a somewhat predictable use of language. It is also put forward by Willis (1996). She suggests a range of task designs that can be applied to texts. Prediction from the previous text is one of them.

In this task the students communicate and cooperate with each other. Each one is responsible for his/her own part and they must work together and check others' work if the paragraph is cohesive. They have to negotiate exchange and share the information and ideas to complete the task. In this way, their ability of using language can be improved. At the same time, the teacher should monitor from a distance to encourage all attempts at communication, not correcting.

Look back to the whole lesson, the aim of the experimental teaching plan is to have the students get the main idea of the text, develop their imagination on the basis of reason and develop their reading skills and further speaking ability. Tasks like sharing personal experiences, skimming and scanning, ordering and reporting in groups, speculating and role-playing in pairs are all used to achieve these goals effectively.

\section{CONCLUSION}

This paper concentrates on the practical aspect of using task-based language teaching ideas in the classroom and conducts a detailed research into the task-based approach. It has covered the following main points: the theories of TBLT; the comparison between TBLT and traditional teaching methods; a research in current situation of reading classroom and its findings; the necessity of the shift to TBLT; a reading lesson plan based on TBLT.

TBLT focuses on how to learn rather than what to learn. The task is a means of using the language in order to learn the language. It has meaning for students who have to solve communication problems, and that meaning, along with the authenticity in the use of real-life situations, becomes internalized as linguistic competence. At last, the process of understanding, performing and reflecting on the task produces a wealth of 'real' use of the target language, all of which foster language learning in cyclical, ongoing manner. Therefore, compared with traditional approaches, these positive results confirm the feasibility of implementing TBA.

Despite the promising aspects of TBA, specific problems may be found in the implementation, which present a diversity of challenges for researchers and English teachers. As a new orientation of communicative language teaching, TBLT requires long-term planning and serious organizational considerations. Nevertheless, the study is promising and it is hoped that there will be more and more teachers and scholars to make theoretical researches in a deep-going way and explore its applications, especially its applications to the improvement of English reading classroom in China.

\section{REFERENCES}

[1] Bygate, M. (2001). Researching Pedagogic Tasks (Applied Linguistics and Language Study). Cambridge: Cambridge University Press.

[2] Gray, K. (1990). Design for the General Class. Oxford: Oxford University Press.

[3] Grellet, F. (1981). Developing Reading Skills. Cambridge: Cambridge University Press.

[4] Hover, D. (1986). Think Twice. Cambridge: Cambridge University Press.

[5] Long, M. (1985). A Role for Instruction in Second Language Acquisition. Clevedon Avon: Multilingual Matters.

[6] McDowell, J., James, P. \& Rich, P. (1984). Basic Information: a task-based approach to developing reading skills at elementary level. London: Edward Arnold.

[7] Nunan, D. (1989). Designing Tasks for The Communicative Classroom. Cambridge: Cambridge University Press.

[8] Prabhu, N. S. (1987). Interactive Language Teaching. Cambridge: Cambridge University Press.

[9] Richards, J. C., Platt, J. \& Weber, H. (1986). Longman Dictionary of Applied Linguistics London. Harlow, U.K.: Longman Addison- Wesley.

[10] Richards, J. \& T. Rodgers. (1986). Approaches and Methods in Language Teaching. Cambridge: Cambridge University Press.

[11] Skehan, P. (1998). A Cognitive Approach to Language Learning. Oxford: Oxford University Press.

[12] Willis, J. (1996). A Frame Work for Task Based Learning. Harlow, U.K.: Longman Addison- Wesley.

Zan Mao was born in Zhenjiang, China in 1985. She received her B.A. degree in Foreign Linguistics from Shanghai Normal University, China in 2007.

She is currently an assistant in English Department, Zhenjiang Watercraft College of PLA, Zhenjiang, China. Her research interests include Second Language Acquisition and Foreign Language Teaching. 